\title{
NEW YORK SOCIETY \\ FOR \\ PSYCHOTHERAPY AND PSYCHOPATHOLOGY MEMBERSHIP LIST
}

Addresses are in New York City unless otherwise stated.

Adams, Earl H., 30 E. 76th St. Ames, Thaddeus H., 55 Park Ave. Amsden, George S., iI 5 E. 67 th St. Barach, Alvan, 893 Park Ave. Bender, Lauretta, 325 E. 4tst St. Berman, William, 204 Martine Ave., White Plains, N. Y.

Berner, Frank, 133 E. 58th St. Blatw, Daniel, New Canaan, Conn. Booth, Gotthard Cohen, 114 E. 54th St. Bowman, Karl M., Rellevue Hospital. Brill, A. A., Is W. 7oth St.

Colbert, Carter B., 307 F.. 44th St. Conrad, Agnes, 59 E. $77_{\text {th }}$ St. Curran, Frank J., 404 E. 55th St. Diethelm, Osxar, 525 E. 68th St. Draper, George, 33 E. 68th St. Dune, William H., 178 E. 7oth St. Fliess, Robert, io2 E. 22nd St. Ginsburg, Sol W., I 2 E. $97^{\text {th }}$ St. Glueck, Bernard, 103 E. 39th St. Goldstein, Kurt, ro E. 85 th St. Goldstein-Rothmann, Eva, 10 E. 85 th St. Greenacre, Phyllis, 345 E. 68th St. Hart, Henry H., 16 F. goth St. Herslof f, Nils B., 47 I Park Ave. Hinkle, Beatrice, 3 I Gramercy Pk. Hinsie, Leland E., 722 W. 168th St. Horney, Karen, 160 Central Park So. Jameison, Gerald R., 525 E. 68th St. KempF, Edward G., Wading River, L. I. Keiser, Sylvan, 50 E. 2 ist St., Brooklyn. KNOPF, Olga, 210 E. 68th St.

LaMar, Norvelle C., I49 E. 73rd St. LEVY, DAVID M., I 45 F., 57 th St.

Lewis, Nolan D. C., 722 W. I68th St. Lipshutz, Danjel M., 22 W. 77 th St.
Liss, Edward, 130 E. 39th St.

Lorand, SANDOR, I 15 E. 86th St.

McGraw, Ronert B., I80 Ft. Wash. Ave. Mayer, Max D., 1192 Park Ave. Millet, Juhn A., 770 Park Ave.

Oberndorf, C. P., 112 Central Park W.

Pot.atte, Philip, 722 W. 168th St.

Powdermaker, Florfince, 52 E. 66th St.

Ross, Nathaniel, 107 W. IIth St.

Rotrengero, Simon, 175 Eastern Parkway, Brooklyn.

Rothschild, Leonard, 745 Fifth Ave.

Ryan, Charles D., 525 E. 68th St.

Sands, Irving, 202 New Yotk Ave., Brooklyn.

Schilder, Paul, 160 E. 48 th St.

Selby, Nathaniel, I 33 E. 58th St.

Sprague, George S., 121 Westchester Ave. White Plains, N. Y.

Strong, A. McIntYre, 180 Fort Washington Ave.

Tallman, Frank P., Rockland State Hospital, Orangeburg, N. Y.

Thomson, Mary M., 34 E. 5oth St.

Tiebout, Harry M., Blythewood, Inc., Greenwich, Conn.

van Ophuijsen, J. H. W., 12 E. 86th St.

Vessie, Percy R., Greenwich, Conn.

WaLL, Jas. H., 12I Westchester Ave,, White Plains, N. Y.

Wechiser, Israel, 70 E. 83rd St.

WENDER, Lours, Hastings-on-Hudson, N. Y.

Wrtrels, Fritz, gi Central Park W.

Wortis, Herman, i7 F. 96th St.

Wortrs, Jos., I 52 Hicks St., Brooklyn.

Wortis, S. Bernard, 410 E. $57^{\text {th }}$ St. 